University of Nebraska - Lincoln

DigitalCommons@University of Nebraska - Lincoln

\title{
Evolutionary Endocrinology: The Developing Synthesis between Endocrinology and Evolutionary Genetics
}

Anthony J. Zera

University of Nebraska - Lincoln, azera1@unl.edu

Lawrence G. Harshman

University of Nebraska - Lincoln, Iharshman1@unl.edu

Tony D. Williams

Simon Fraser University

Follow this and additional works at: https://digitalcommons.unl.edu/bioscizera

Part of the Microbiology Commons

Zera, Anthony J.; Harshman, Lawrence G.; and Williams, Tony D., "Evolutionary Endocrinology: The Developing Synthesis between Endocrinology and Evolutionary Genetics" (2007). Anthony Zera Publications. 40.

https://digitalcommons.unl.edu/bioscizera/40

This Article is brought to you for free and open access by the Papers in the Biological Sciences at DigitalCommons@University of Nebraska - Lincoln. It has been accepted for inclusion in Anthony Zera Publications by an authorized administrator of DigitalCommons@University of Nebraska - Lincoln. 
Published in Annual Review of Ecology, Evolution, and Systematics 38 (2007), pp. 793-817; doi: 10.1146/annurev.ecolsys.38.091206.095615 Copyright (c) 2007 Annual Reviews Inc. Used by permission.

Published online August 20, 2007

\title{
Evolutionary Endocrinology: The Developing Synthesis between Endocrinology and Evolutionary Genetics
}

\author{
Anthony J. Zera, ${ }^{1}$ Lawrence G. Harshman, ${ }^{1}$ and Tony D. Williams ${ }^{2}$ \\ ${ }^{1}$ School of Biological Sciences, \\ University of Nebraska-Lincoln, Lincoln, NE 68588; \\ email azera1@unl.edu,lharsh@unl.edu \\ ${ }^{2}$ Department of Biological Sciences, \\ Simon Fraser University, Burnaby, British Columbia, Canada V5A 1S6; \\ email tdwillia@sfu.ca
}

\begin{abstract}
A productive synthesis of endocrinology and evolutionary genetics has occurred during the past two decades, resulting in the first direct documentation of genetic variation and correlation for endocrine regulators in nondomesticated animals. In a number of insect genetic polymorphisms (dispersal polymorphism in crickets, butterfly wing-pattern polymorphism), blood levels of ecdysteroids and juvenile hormone covary with morphology, development, and life history. Genetic variation in insulin signaling may underlie life history trade-offs in Drosophila. Vertebrate studies identified variation in brain neurohormones, bone-regulating hormones, and hormone receptor gene sequences that underlie ecologically important genetic polymorphisms. Most work to date has focused on genetically variable titers (concentrations) of circulating hormones and the activities of titer regulators. Continued progress will require greater integration among (a) traditional comparative endocrine approaches (e.g., titer measures); (b) molecular studies of hormone receptors and intracellular signaling pathways; and (c) fitness studies of genetically variable endocrine traits in ecologically appropriate conditions.
\end{abstract}




\section{Introduction}

The evolution of endocrine regulation has been a topic of interest to physiologists and evolutionary biologists for decades (reviewed in Adkins-Regan 2005, Matsuda 1987, Nijhout \& Wheeler 1982, Norris 1997, West-Eberhard 2003, Zera 2004). Major issues in this topic include the nature of endocrine adaptations (i.e., evolutionary modifications of endocrine control mechanisms) and the role of these modifications in the evolution of morphology, behavior, development, and life history. Until fairly recently, the vast majority of evolutionary studies focused on comparative aspects of hormonal regulation among species or higher taxa. Typical examples include phylogenetic analyses of hormone structure/function (Colon \& Larhammar 2005, Guilgur et al. 2006, Norris 1997) or comparative studies of various endocrine adaptations (e.g., differences in the hormonal control of ion balance among terrestrial, fresh-water, and salt-water vertebrates; reviewed in Norris 1997). Another important research focus has been the hormonal control of phenotypic trade-offs among life history traits in the field, such as testosterone-mediated trade-offs; (Ketterson et al. 2001, Marler 1988, Svensson et al. 2002). However, microevolutionary investigations of genetically variable hormonal regulators in nondomesticated animals were rare before 1990 (Kallman 1989, Zera \& Teibel 1989).

This situation has changed dramatically, and the first substantial synthesis of endocrinology and evolutionary genetics, termed evolutionary endocrinology (Zera \& Huang 1999), has begun. There are two main reasons for this development: First and foremost, during the past 20 years, evolutionary biology has focused increasingly on the microevolution and evolutionary genetics of complex organismal adaptations, such as life history traits (fecundity, longevity), life history trade-offs, and aspects of development (evolution of butterfly wing patterns, dispersal polymorphisms, body size). Hormones regulate most major components of development and life history, such as growth rate, body size, timing of metamorphosis, and sexual maturation. Furthermore, individual hormones typically affect numerous phenotypes (endocrine pleiotropy) and thus almost certainly give rise to genetic correlations that underlie constraints and trade-offs; these correlations strongly influence the evolution of development and life histories. A growing consensus has emerged that a detailed understanding of the microevolution of life histories and development requires a corresponding deep understanding of the microevolution of the endocrine mechanisms that control the expression of these traits (Brakefield et al. 2003, Finch \& Rose 1995, Harshman \& Zera 2007, West-Eberhard, 2003, Zera \& Harshman 2001). Second, the recent development of evolutionary physiology (Feder et al. 1987, Garland \& Carter 1994) highlighted the importance of investigating within-species genetic variation and covariation for physiological processes to understand the mechanisms by which physiology evolves. Thus, evolutionary-genetic analysis of within-population hormonal variation is increasingly viewed as an important research focus in its own right (Zera 2006, Zera \& Huang 1999, Zera \& Zhang 1995).

This review deals exclusively with animals, primarily insects and vertebrates, groups in which most evolutionary-endocrine studies have been conducted. We focus primarily on the following two topics: $(a)$ the extent and nature of genetic varia- 
tion and covariation for various endocrine traits and $(b)$ the extent to which endocrine regulators (e.g., hormone levels, regulators of hormone titers, hormone receptor expression) covary genetically with whole-organism aspects of morphology, development, and life history, and the evolutionary implications of these associations. Our primary goal is to evaluate critically the most significant experimental findings in evolutionary endocrinology from the past two decades.

\section{Background in Evolutionary Endocrinology}

\section{A Thumbnail Sketch of Endocrine Regulation}

The mechanisms that comprise endocrine regulation are exceedingly complex, and we give only a brief outline of the main aspects here to aid the nonspecialist. For general reviews see, Adkins-Regan 2005, Gilbert et al. 2005, Kacsoh 2000, Nijhout 1994, and Norris 1997. The key components of endocrine signaling are (a) the systemic (e.g., blood) concentration or titer of a hormone (the signal), (b) the receptors that bind the hormone (the receiver; tissue sensitivity), and (c) the various intracellular mechanisms that convert hormone binding into production of effector molecules (e.g., enzymes; regulatory molecules). A hormone can be either the direct product of a gene (e.g., peptide hormones such as insulin and insulin-like growth factors), or a molecule that is the end product of a biosynthetic pathway (e.g., steroids such as testosterone and ecdysone). Hormones or prohormones are secreted into the circulatory system and may be modified (e.g., activated) in various tissues; hormones are transported either unbound (many peptide hormones) or bound to a carrier protein (many lipophilic hormones) to target tissues. The hormone can then interact with the outer cell membrane (e.g., polar peptide hormone binds to receptor), which initiates an often complex intracellular signaling cascade. Alternatively the hormone can enter the cell (e.g., steroid), where it often binds to a receptor protein and is transported to the nucleus where it modulates gene transcription. The hormone is then degraded and/ or excreted.

A hallmark of hormonal regulation is the complex network of interactions that underlies such key phenomena as hormonal homeostasis, hormonal pleiotropy, and phenotypic integration. For example, the blood titers of many hormones (e.g., testosterone, estrogen, and ecdysone) are often maintained within a narrow range (homeostasis) by multiple negative feedback loops between the hormones and the neurohormonal regulators that control hormonal secretion. Similarly, the regulation of phenotypic expression by a specific hormone often requires the prior action of other hormones (priming effects) and modulation by additional hormones. Thus, the endocrine control of complex phenotypes, such as the timing of metamorphosis or egg production, typically requires a precisely orchestrated sequential production and elimination of numerous interacting hormones. Finally, a single hormone often influences the expression of multiple phenotypes (hormonal pleiotropy), which is a key aspect of the integrative expression of multiple components of a complex adaptation (phenotypic integration). These complex interactions can make the analysis of endocrine regulation exceedingly difficult. 


\section{Studied Endocrine Traits and Endocrine Methods}

Of the three main components of endocrine regulation discussed above, thus far hormone titer has been studied most extensively from an evolutionary-endocrine perspective. This predominant focus on hormone titers is the consequence of a large body of evidence that implicates the regulation of phenotypic expression by changes in circulating hormone levels, such as the hormonal regulation of developmental and reproductive aspects of insect and vertebrate polymorphisms (Hartfelder \& Emlen 2005, Nijhout 1994, Shi 2000). Moreover, specific and sensitive assays, such as radioimmunoassays, have long been available, which allow the measurement of hormone levels in the relatively large number of individuals often required in quantitative-genetic studies of endocrine variation (Zera \& Cisper 2001, Zera \& Zhang 1995, Zijlstra et al. 2004). Conversely, the effective titer available to tissue receptors may not necessarily correspond to the total blood hormone titer. For example, binding by plasma proteins can modulate the effective titer of a hormone (e.g., corticosterone; Breuner \& Orchinik 2002), as can proximity to the site of hormone production/release (e.g., the concentration of testosterone, which is required for proper sperm production, is much higher in the testes than in the blood) (Kacsoh 2000). No evolutionary-endocrine studies (and few ecological/behavioral studies) have investigated these more complex aspects of hormone titers.

Some of the most detailed evolutionary-endocrine studies have focused on general regulators of the hormone titers, most notably, the activities of hormone-degrading enzymes (Zera 2006, Zera \& Huang 1999). These endocrine traits are often easier to measure than hormone titers or receptor characteristics, often play an important role in hormone titer regulation, and can be measured in small individual organisms (e.g., insects), without severe damage, which is an important advantage in artificial selection studies (Zera \& Zhang 1995).

Intraspecific variation in receptor attributes (e.g., tissue or stage-specific receptor-gene expression and hormone-binding affinity) may play an important role in endocrine adaptation. Receptor alterations can result in phenotypic changes that are restricted to the specific tissue or organ in which they occur, in contrast to the widespread phenotypic (i.e., pleiotropic) alterations expected to occur when the systemic titer of a hormone is changed. In some cases, such as for juvenile hormones, the receptor has not been unambiguously identified, thus precluding serious study of intraspecific variation in tissue sensitivity for this important group of hormones. Although researchers have investigated receptors extensively in comparative endocrine studies of vertebrates (Norris 1997), functional studies of receptor variation are rare in evolutionary-endocrine studies as well as in field-ecological studies (for an exception see Hoekstra et al. 2006, discussed in Vertebrate Studies, below).

The paucity of studies on receptors and components of intracellular signaling pathways testifies to the nascent state of evolutionary endocrinology. However, this situation almost certainly will change dramatically in the next decade. Researchers have undertaken extensive mutational/molecular analyses of signaling pathways and hormone receptors in a few model genetic organisms such as Drosophila, C. elegans, and mice (Harshman \& Zera 2007, Henrich 2005, Partridge et al. 2005). These 
model organisms and molecular approaches are beginning to be incorporated into evolutionary-endocrine research at an increasing rate (Harshman \& Zera 2007, Richard et al. 2005); a number of recent, notable examples are discussed throughout this review.

The identification of fitness effects of endocrine variation is also a key aspect of evolutionary endocrinology. This remains to be done for endocrine genotypes, although several detailed studies in vertebrates addressed this issue at the phenotypic level (discussed below in Vertebrate Studies).

The misuse of hormone manipulation. One widespread and growing problem in evolutionary endocrinology, which deserves special comment, is the inappropriate use of hormone manipulation (HM), which involves experimental manipulation of the in vivo titer of a hormone [see Zera (2007b) for a detailed discussion of this topic]. HM is very useful in evolutionary endocrinology, for example, to evaluate the functional significance of hormone-phenotype correlations identified in genetic analyses (Zijlstra et al. 2004; see Genetic Polymorphism for Wing Pattern and Life History in Bicyclus, below), or to produce phenotypes for experiments (e.g., Ketterson et al. 2001; see Vertebrate Studies, below). However, HM has been commonly misused; it should not be the sole or primary empirical method used to establish the role of a hormone in the regulation of phenotypic expression (e.g., the juvenile hormone studies of Emlen \& Nijhout 1999, 2001; Meylan \& Clobert 2005; for a detailed critique see Zera 2007b). By itself, HM can never provide more than weak support for the role of a particular hormone or its specific mechanisms of action in the regulation of phenotypic expression; applied hormones or analogs can have strong pharmacological (nonphysiological) effects, especially when applied in nonphysiological concentrations (which is often the case in insect studies) or at inappropriate times in the life cycle. Furthermore, introduced hormones can alter titers or receptors of other hormones. If variation in an endocrine trait is the focus of study, that endocrine trait should be quantified directly via the use of a well-validated assay, no matter how laborious.

\section{Genetic Approaches in Evolutionary Endocrinology}

Most evolutionary-endocrine studies to date focused on physiological aspects of hormonal variation such as hormone titers or activities of hormone-regulating enzymes, via the use of quantitative-genetic methods, the most common of which is artificial selection. For general reviews of artificial selection see Gibbs (1999), Harshman \& Hoffmann (2000), Swallow \& Garland (2005), and Falconer \& MacKay (1996). The degree of response to artificial selection quantifies the genetic component of phenotypic variation for the selected trait (heritability) (Zera \& Zhang 1995). Researchers identify endocrine factors that potentially regulate the expression of morphological/ life history traits as indirect (correlated) responses when nonendocrine phenotypes (e.g., life history traits) are selected directly [Malisch et al. 2007, Zera 2006, Zera \& Huang 1999, Zijlstra et al. 2004; see Discontinuous Variation (Genetic Polymorphism) in Insects, below]. 
A major advantage of artificial selection is the production of genetically differentiated populations for the selected and correlated phenotypes; these stocks can then be subjected to further analyses to identify the underlying causes of phenotypic divergence and correlations. For example, researchers used this approach to investigate the molecular and biochemical causes of line differences in the activity of the cricket juvenile hormone titer regulator, juvenile hormone esterase (JHE) (Zera 2006; discussed below in the section on Direct Selection on an Endocrine Regulator in Gryllus assimilis). Individual genes that contribute to physiological-genetic variation in an endocrine trait or a phenotype controlled by that regulator can also be identified in artificially selected lines via the use of standard quantitative-genetic methodologies, such as quantitative trait locus (QTL) mapping or the investigation of candidate genes. For example, QTL analysis of variation in the corticosterone-mediated stress axis in the rat identified a complex genetic architecture consisting of multiple interacting genetic factors with maternal and sex effects (e.g., Solberg et al. 2006). Until very recently, this approach was not often used in evolutionary-endocrine studies, and contributions of individual genes to a quantitative endocrine trait of interest are not well known [however see Crone et al. (2007) and Hoekstra et al. (2006); discussed below in the sections on Continuous Variation in Insects and on Vertebrate Studies]. We expect such analyses to become common in the next decade.

Quantitative-genetic studies of endocrine variation have been conducted with varying degrees of rigor. For example, some studies used full-sib breeding designs or regression of offspring on the female parent, which can significantly inflate heritabilities for a variety of reasons (e.g., maternal effects; King et al. 2004); many of these problems can be circumvented by half-sib analyses (Gu \& Zera 1996; Roff et al. 1997). In some artificial selection studies (Suzuki \& Nijhout 2006, Zera et al. 1989), researchers selected only one line in a particular direction (nonreplicated selection). Only suggestive genetic inferences, such as the existence of genetic differences between lines or genetic correlation, can be drawn from unreplicated studies (Falconer \& Mackay 1996) without additional experiments such as line crosses (as in Zera \& Teibel 1989).

Assessment of the functional significance of genetic correlation between a hormonal trait (e.g., hormone titer) and a phenotype (e.g., morphological trait) also requires care because correlation does not necessarily imply causation. Spurious, nonfunctional endocrine correlations may result from the extensive cross talk between hormonal regulators (see A Thumbnail Sketch of Endocrine Regulation, above). The identification of such correlations may be difficult but it is critically important and will typically require experimental manipulation; in this context, HM can be a powerful experimental technique (Zijlstra et al. 2004; see The Misuse of Hormone Manipulation, above). Researchers have substantially underestimated the difficulty involved in assessing the functional significance of endocrine correlations in many evolutionary-endocrine studies.

Mutational analysis is a more recent genetic approach in evolutionary endocrinology and is becoming more common. This approach uses laboratory-generated single-locus mutations, typically of large effect, to identify hormonal traits (e.g., components of signaling pathways) that control the expression of complex phenotypes. An example of this approach is the use of mutations in the insulin signaling pathway to 
identify the role of this pathway in life history variation and trade-offs in Drosophila (Flatt et al. 2005, Richard et al. 2005). Currently, mutational analysis is restricted almost exclusively to model genetic organisms (e.g., C. elegans, Drosophila). The great power of mutational analysis is its ability to dissect the mechanisms of hormone action on ecologically important traits by identifying the influence of often well-characterized individual endocrine loci on a phenotype of interest. The limitation of this approach is that mutations of large effect, which are typically used in mutational analysis, often do not segregate in natural populations. Thus, mechanisms that underlie the hormonal control of phenotypic variation identified by mutational analysis may be different from the mechanisms in natural or laboratory populations (Brakefield et al. 2003, Harshman \& Zera 2007, Stern 2000). Relatively little is known currently about the nature of allelic variation in endocrine system genes that segregate in populations. A few notable physiological studies focused on spontaneous, singlelocus endocrine mutations in the laboratory (Rountree \& Nijhout, 1995a,b; Suzuki \& Nijhout 2006); a spate of recent molecular studies investigated single-locus (or oligogenic) polymorphisms in natural populations of vertebrates (Colosimo et al. 2005, Hoekstra et al. 2006, Mundy et al. 2003, Nachman et al. 2003; all discussed below in Vertebrate Studies).

\section{Empirical Studies}

\section{Discontinuous Variation (Genetic Polymorphism) in Insects}

Wing (dispersal) polymorphism in crickets. During the past 15 years, investigators studied, from an endocrine-genetic perspective, a number of complex (multitrait) polymorphisms that play an important role in the life cycle of the organism. Most notable are dispersal-polymorphism in crickets and wing-pattern polyphenism in butterflies (reviewed in Brakefield \& Frankino 2007; Brakefield et al. 2003; Zera 2004, 2006). Polymorphism is defined here as discontinuous variation in some phenotype. If phenotypic variation results from variation in genotype, it is termed genetic polymorphism; if phenotypic variation is due to environmental variation, it is termed polyphenism.

Wing polymorphism in species of Gryllus (crickets) has been especially well-studied with respect to systemic variation in the titers of juvenile hormone $(\mathrm{JH})$ and ecdysteroids, two key developmental and reproductive hormones, and the role of titer variation in regulating aspects of development (such as alternate morph production) and adult-life history (e.g., the trade-off between flight capability and reproduction). Wing polymorphism in Gryllus is both a genetic polymorphism (polygenic, with a threshold) (Roff, 1996) and an environmental polyphenism (Zera 2004, 2007a). However, virtually all endocrine work on this model focuses on genetic polymorphism, and employs artificial selection in a single laboratory environment. The polymorphism consists of a flight-capable morph that has long wings and large flight muscles, but delays egg production and has low fecundity. The alternate, flightless morph has the converse set of traits (Zera 2004, 2006, 2007a). In two Gryllus species, using field- 
collected individuals, researchers obtained selected lines that produced $>90 \%$ of the dispersing or flightless/reproductive morph; these selected lines were subjected to endocrine analyses (reviewed in Zera 2004, 2006, 2007a).

In G. rubens and G. firmus, lines selected for the dispersing morph exhibited (a) much higher blood activity of the enzyme JHE, which degrades $\mathrm{JH},(b)$ a reduced $\mathrm{JH}$ titer, and $(c)$ an elevated ecdysteroid titer compared with lines selected for the flightless/reproductive morph. These differences occurred during a critical period in morph development. High JHE activity strongly cosegregated with the dispersing morph in crosses and backcrosses of selected populations of G. rubens, which is the first demonstration of genetic covariance (i.e., cosegregation) between a variable endocrine and developmental/life history trait in insects recently derived from the field. Because ecdysteroids promote growth and differentiation, whereas JH inhibits metamorphosis, the higher ecdysteroid titer and lower JH titer (and higher JHE activity) are consistent with a role for either or both of these hormones in the regulation of morph development (Zera 2006). In contrast, JH binding in the hemolymph (due to the JH binding protein) did not differ between morphs (Zera \& Holtmeier, 1992), indicating that variation in JH binding does not likely play a major role in the endocrine regulation of morph development. Zera and coworkers have also undertaken one of the few direct comparisons between genetic polymorphism and environmental polyphenism for an endocrine trait, JHE activity (Zera 2006, Zera \& Teibel 1989; also see Rountree \& Nijhout 1995a,b and Suzuki \& Nijhout 2006).

Endocrine studies of Gryllus have a number of limitations. For example, despite considerable study, the relative importance of $\mathrm{JH}$ versus ecdysteroids (or other hormones) in the regulation of aspects of morph expression still remains unresolved, and $\mathrm{JH}$ titer differences between morphs are not large (see extensive discussion in Zera 2004, 2006). Because a nuclear JH receptor has not been unequivocally identified in insects, the extent to which morph-specific traits result from variation in receptor characteristics, as opposed to variation in circulating hormone levels, cannot be assessed. Finally, the influence of hormones other than JH or ecdysteroids on morph development, or the endocrine mechanisms that coordinate morph development with morph-specific reproduction in adults, are unknown (Zera 2004, 2006).

Because JH plays an important role in insect reproduction (Gilbert et al. 2005, Nijhout 1994), endocrine-evolutionary models of complex polymorphism have long proposed that the blood JH titer should be higher in the morph with elevated fecundity. However, this hypothesis has been tested directly in dispersal-polymorphic species only during the past few years (Zera 2004, 2006). Surprisingly, in G. firmus the JH titer in the dispersing morph exhibits a high amplitude, circadian rhythm (50-fold titer change over a 6 hour period) that cycles above and below the relatively invariant titer in the flightless morph (Zhao \& Zera 2004). These data suggest that morph-specific effects of JH may be determined not only by the titer, but also by the length of time during which the titer is elevated above a threshold (Zera et al. 2007). By contrast, the ecdysteroid titer is consistently elevated in flightless versus dispersing females and may contribute to the elevated egg production of the flightless morph in concert with or independent of JH. The unexpected morph-specific JH circadian rhythm illustrates the importance of measuring hormone titers directly over short timescales. A num- 
ber of studies recently showed phenotypic variation in circadian or diurnal rhythms for endocrine traits in insects (Vafopoulou \& Steel 2005; Zhao \& Zera 2004). The failure to identify these endocrine titer rhythms, especially if the rhythms are morphspecific, can result in substantial errors in interpretation of the endocrine data (Zera 2006, 2007b).

Although field-endocrine studies are common in vertebrates (discussed in Vertebrate Studies, below), only a few limited studies have been undertaken in insects (reviewed in Zera et al. 2007). Thus, the correspondence between endocrine traits measured in selected lines in the laboratory and measured under field conditions is poorly understood. However, a recent multi-year field study found comparable results for blood JH and ecdysteroid titers in G. firmus in individuals sampled in the field or in selected lines raised under field conditions, as were found in laboratory studies described in the paragraph above (Zera et al. 2007; and A.J. Zera, unpublished observations). The morph-specific circadian rhythm for JH titer in field populations of G. firmus is a powerful model with which to investigate the microevolution of endocrine circadian rhythms, a poorly studied topic in evolutionary endocrinology.

Genetic polymorphism for wing pattern and life history in Bicylcus. Butterfly wing pattern components (e.g., size, color, shape) are exquisite adaptations for camouflage, thermoregulation, mate recognition, etc. Developmental, hormonal, biochemical, and more recently, molecular aspects of variation in wing pattern expression have been studied extensively, most often in the context of seasonal polyphenism (reviewed in Brakefield \& Frankino 2007, Nijhout 1991). Various neuropeptides (Jones et al. 2006) and ecdysteroids are implicated as regulators of pigment biosynthesis and eyespot patterning.

The most detailed endocrine-genetic analyses of wing-pattern components are studies of ecdysteroid control of eyespot size and life history (rate of development) in the butterfly Bicyclus anyana (Brakefield \& Frankino 2007, Koch et al. 1996, Zijlstra et al. 2004). Size of the ventral eyespot and rate of development are adaptations to a particular season (polyphenism) (Brakefield \& Frankino 2007). Researchers conducted artificial selection in the laboratory in an environment intermediate between those producing the seasonal phenotypes, and on a base population that contained ventral eyespot sizes intermediate between those seen in wet and dry seasons. Selection on ventral eyespot size or rate of development demonstrated that these characteristics are correlated genetically with each other and with the hemolymph ecdysteroid titer during early pupal development. Results of ecdysteroid injection experiments implicated an earlier rise in the ecdysteroid titer as a cause of increased ventral eyespot size and faster rate of development in the wet-season morph, although a more complex picture emerged subsequently (Zijlstra et al. 2004).

Simultaneous two-trait selection on ventral eyespot size and rate of development produced four lines with the four possible trait combinations (fast-development rate and large eyespots, slow-development rate and large eyespots, etc.). Unexpectedly, investigators observed genetic covariance between the ecdysteroid titer and development rate, but not ventral eyespot size (Zijlstra et al. 2004). Nevertheless, injection of ecdysteroids increased eyespot size as well as rate of development, as observed pre- 
viously (Koch et al. 1996). A confounding problem with hormone injection was the strong effect of injection per se on eyespot size (see Zijlstra et al. 2004). These authors concluded that the ecdysteroid titer primarily controls development rate, whereas ventral eyespot size can be modulated by ecdysteroid-dependent as well as ecdysteroid-independent mechanisms. These important studies illustrate that $(a)$ endocrinegenetic control mechanisms can be more complex than they appear at first, and $(b)$ the initial demonstration of hormone-phenotype associations should be viewed as the beginning, not the end point, of functional studies of endocrine control of phenotypic expression.

Evolution of a polyphenism by artificial selection. Although numerous studies investigated the mechanisms that underlie morph expression in polyphenisms, little is known about the evolutionary origins of polyphenism. In an innovative study, Suzuki \& Nijhout (2006) addressed this issue with direct selection for plasticity in body color in Manduca sexta, a model organism in insect endocrine studies. The authors heat shocked the Manduca sexta black mutant to expose hidden genetic variation in coloration, which provided the means for selection. The authors produced a line (unreplicated) whose body color was always black irrespective of rearing temperature (monophenic line), as opposed to another line that was black or green depending on rearing temperature. Owing to the extensive endocrine database for this model species (Nijhout 1994), Suzuki \& Nijhout (2006) could investigate the endocrine basis of the evolution of plasticity. These authors reported that the plastic line evolved by selection on genes that regulate temperature-dependent hemolymph juvenile hormone titer. One limitation of this study is that Suzuki \& Nijhout (2006) used a bioassay to measure the JH titer; bioassays can be unreliable (see Baker 1990, Zera 2007b).

Other polymorphisms/polyphenisms. A number of other groups investigated JH regulation of complex polymorphism, such as horn size polymorphism in dung beetles and wing polymorphism in the soapberry bug (Dingle \& Winchell 1997; Emlen \& Nijhout 1999, 2001; Moczek \& Nijhout 2002). Unfortunately, in these studies hormone manipulation (HM) was the only endocrine technique used by researchers to investigate $\mathrm{JH}$ regulation; they failed to measure any aspect of $\mathrm{JH}$ signaling directly, such as JH titer or activities of JH titer regulators [see Zera (2007b) for a detailed critique]. Conversely, preliminary data suggest ecdysteroid titer differences between nascent horn morphs (Emlen \& Nijhout 1999). Rountree \& Nijhout (1995a,b) investigated the ecdysteroid regulation of wing color pattern in the butterfly Precis coenia in natural polyphenic morphs and in a spontaneous laboratory mutant.

\section{Continuous Variation in Insects}

Direct selection on an endocrine regulator in Gryllus assimilis. The activity of the hormone-regulating enzyme JHE in the field cricket Gryllus assimilis was the first endocrine trait directly subjected to artificial selection in a laboratory population recently founded from field-collected individuals (Zera \& Zhang 1995). These studies combined with other quantitative-genetic analyses (Gu \& Zera 1996) and studies of 
JHE activity in wing-polymorphic crickets (discussed above) make JHE the most intensively studied endocrine trait from an evolutionary-genetic perspective (reviewed in Zera 2006).

Researchers observed strong responses to replicated selection in either juvenile or adult stages; heritabilities were similar to those of nonendocrine enzymes (Zera 2006, Zera \& Zhang 1995, Zera et al. 1998). Selection on JHE activity during the juvenile or adult stages resulted in no correlated responses on JHE activity during the alternate life cycle stage. These results demonstrate that some components of endocrine regulation can evolve independently in a stage-specific manner. The extent to which physiological mechanisms constrain various life cycle stages to evolve in concert is a key but poorly understood topic in evolution (Brakefield et al. 2003, West-Eberhard 2003, Zera 2006). Extensive studies of morphological, developmental, physiological, biochemical, and molecular correlates of JHE activity in selected lines of G. assimilis were reviewed recently in Zera (2006), and only more recent or salient findings are covered here.

Characterizations of selected lines of G. assimilis show that the response to divergent selection on blood JHE activity results equally from genes that contribute to: (a) whole-organism enzyme activity and $(b)$ the degree of enzyme secretion into the blood, similar to G. firmus (Zera \& Huang, 1999). Enzymatic properties do not differ between JHEs from high and low activity lines, nor are there nucleotide differences in the coding sequence of JHE genes within or between selected lines (Crone et al. 2007 and references therein). Cosegregation between line-specific JHE allele and blood JHE activity implicates DNA sequence variation at or near the JHE gene region as a contributor to line differences in blood JHE activity. The study by Crone and coworkers (2007) is one of the few that links molecular aspects of a candidate gene to physiological/phenotypic variation in an endocrine trait in an insect (analogous studies in vertebrates are discussed in the sections on Vertebrate Studies and on Single Locus Polymorphisms in the Field).

Comparisons among the results of artificial selection studies performed in G. rubens, G. firmus, and G. assimilis provide important insights into $(a)$ the nature of the genetic factors responsible for microevolutionary changes in JHE activity, $(b)$ the degree of change in JHE activity necessary to alter whole-organism JH metabolism (a prerequisite for affecting the expression of whole-organism traits), and (c) the role of modulation of JHE activity on the expression of specific phenotypes such as wing length and flight-muscle mass. Each of these issues is discussed in detail in Zera (2006).

Body size in insects. Body size is an important organismal trait (reviewed in Edgar 2006, Nijhout 2003). There has been a recent surge of molecular-endocrine studies on body size regulation in Drosophila, whereas physiological-hormonal investigations of body size regulation in M. sexta have been ongoing since the 1970s. These studies provided the impetus for the first evolutionary-endocrine studies of body size in insects.

Nijhout and colleagues (2003) developed a physiological model that relates attainment of size at metamorphosis (size when feeding ends in the last larval stage adult size) to various whole-organism growth parameters, which are in turn related to un- 
derlying endocrine events (D'Amico et al. 2001, Edgar 2006, Nijhout 2003). Ninety percent of growth occurs during the last juvenile instar of $M$. sexta; when a genotypespecific critical weight is reached, a series of endocrine events are set in motion, leading ultimately to the cessation of feeding and onset of metamorphosis. The duration of time between attainment of critical weight and cessation of feeding [the interval to cessation of growth (ICG), when most weight gain occurs] is thought to be determined by the rate of decrease in the JH titer, subsequent secretion of the brain neurohormone (PTTH; secretion inhibited by JH), and release of ecdysteroids (release induced by PTTH); ecdysteroid release is a proximate initiator of molting (end of ICG) [see Nijhout (2003)].

Using this model, D'Amico and coworkers (2001) reported that a laboratory population of $M$. sexta evolved $50 \%$ higher body size during a 30 -year period by altering growth rate, duration of ICG [i.e., purported timing of prothoracicotropic hormone (PTTH) release], and critical weight. Davidowitz \& Nijhout (2004) identified how variation in growth rate and ICG interact to produce larger individuals at higher temperatures (thermal reaction norms). Preliminary studies using a full-sib design identified genetic variation for various size parameters (Davidowicz et al. 2003) and an extensive artificial selection study has been conducted (G. Davidowicz, personal communication).

The aforementioned Manduca studies are problematic regarding some endocrine aspects. For example, the timing of PTTH release and its functional relationship to the end of ICG is a major aspect of the model (e.g., see figure 3 of D' Amico et al. 2001 and Davidowicz et al. 2003). However, Nijhout and colleagues never measured PTTH release (i.e., PTTH titer) directly in any study that focused on the physiological control of body size; rather, they inferred PTTH release from the appearance of a morphological marker that is thought to be correlated with release of this neurohormone (e.g., see Methods section of D'Amico et al. 2001). However, the correlation has never been directly established, but instead was indirectly inferred in the 1970s on the basis of a best guess as to the timing of PTTH release during the last juvenile instar (Nijhout \& Williams 1974 and references therein). PTTH release (measured directly in several moth species; Rybczynski 2005) is now known to be much more complex than suspected previously.

In Drosophila, recent studies have begun to unravel the detailed mechanisms by which various phylogentically conserved signaling pathways [most notably insulin/ insulin-like growth factors and target of rapamycin (TOR)] influence body size [for a detailed review see Edgar (2006)]. Considerable discussion and empirical work is in progress on alterations in the signaling pathways described above in the context of the evolution of body size or size of organs (e.g., beetle horns) within and between species (De Jong \& Bochdanovits 2003, Emlen et al. 2006, Shingleton et al. 2005).

Life history variation and trade-offs in Drosophila. The use of laboratory-generated mutants is a relatively new approach in evolutionary endocrinology that was pioneered in studies of the hormonal underpinnings of life history variation and trade-offs in D. melanogaster. The endocrine foci of these studies are insulin signaling, juvenile hormones, and ecdysteroids (Clancy et al. 2001, Richard et al. 2005, 
Tatar et al. 2001, Tu et al. 2005). A detailed discussion of these studies, some of which report contradictory findings, is useful to illustrate important issues regarding JH endocrinology in Drosophila and the use of mutational analysis. Laboratoryinduced mutations of $D$. melanogaster that interfere with insulin signaling produce phenotypes that include small size, sterility, and extended life span (Bohni et al. 1999, Clancy et al. 2001, Tatar et al. 2001). Working with Drosophila insulin receptor (DInR) mutations, Tatar and coworkers (2001) proposed that these effects were caused by a defect in the cellular insulin receptor, which resulted in reduced systemic (e.g., circulating) levels of juvenile hormone. In other words, the phenotypic effects of insulin-signaling mutations were proposed to be nonautonomous, that is, not confined to cells with the insulin signaling defect. The key observation for this argument was that $D I n R$ mutations resulted in an approximately $75 \%$ decrease in biosynthesis of juvenile hormone in vitro, relative to wild type. Aerosol exposure of this mutant combination to methoprene, a juvenile hormone analog, rescued egg production, albeit at low levels, and the extended life span phenotype was lost. However, it remains unclear to what degree the rate of in vitro JH biosynthesis in Drosophila mutants and wild type corresponds to levels of these hormones in the body, especially levels of circulating hormones in the hemolymph. In fact, the circulating concentration of juvenile hormones remains unreported in the hemolymph. Preliminary analysis of the hemolymph of feeding last instar D. melanogaster larvae identified methyl farnesoate and bisepoxy JHIII as the two primary JH-like compounds (Jones \& Jones 2007).

Another study in D. melanogaster that used a different mutation of insulin signaling (chico ${ }^{1}$, an insulin substrate protein mutation) also found increased life span (Clancy et al. 2001). Tu and coworkers $(2002,2005)$ reported a reduced rate of JH biosynthesis and a reduced release of ecdysteroids from the ovary in vitro in $c h i c{ }^{1}$, whereas Richard and coworkers (2005) found no reduction in either the rate of JH biosynthesis or the release of ecdysteroids from the ovary in this mutant in a different genetic background. In addition, the DInR mutation exhibited a reduced rate of ecdysteroid release by the ovaries in vitro (Tu et al. 2002). Most importantly, the blood level of ecdysteroids was not lower in the long-lived chico ${ }^{1}$ mutation compared with the other genotypes (Richard et al. 2005). This is the only case in which circulating levels of any hormone were measured directly in an insulin signaling mutation of D. melanogaster. Exposure to methoprene by topical or aerosol application across a range of doses failed to recover egg production in females homozygous for this mutation (Richard et al. 2005). These workers performed a critical experiment to determine whether female sterility is caused by a defect in insulin signaling that is autonomous to the egg or caused by a nonautonomous effect of the lowered level of circulating $\mathrm{JH}$ (or a change in some other systemic factor). They reciprocally transplanted immature ovaries between wild-type and chico ${ }^{1}$ mutants; wild-type ovaries produced mature eggs in chico ${ }^{1}$ females, whereas the $c h i c o^{1}$ ovaries did not mature in wild-type females. Therefore, Richard and coworkers (2005) determined that the effect of the chico ${ }^{1}$ mutation on egg maturation was ovary-autonomous [see also Drummond-Barbaosa \& Spradling (2001)] in the presence of approximately wild-type levels of juvenile hormone biosynthesis and ecdsyteroids in the blood. 
In conclusion, whereas genetic alteration of insulin signaling clearly affects lifespan in D. melanogaster, the influence of JH remains an open question. The answer to the question of whether variation in JH leads to variation in aging may depend on the development of a genetic lesion that can perturb levels of the hormone and development of the capability to measure JHs in the blood (Jones \& Jones 2007). Major differences between studies (Tatar et al. 2001, and Tu et al. 2002, 2005 compared with Richard et al. 2005) remain unresolved and may be due to specific mutations in the insulin signaling pathway, genetic background, or differences in assays. Clearly there are problems with mutation analysis, such as different patterns of pleiotropic effects associated with different mutations or differences in genetic background, but in the long run this methodology will be powerful for the investigation of the effects of hormone variation on life histories and other complex traits.

\section{Vertebrate Studies}

Vertebrate research has provided especially important contributions to evolutionary endocrinology in two areas: (a) physiological and molecular aspects of single-locus endocrine polymorphisms that occur in the field and $(b)$ fitness consequences of endocrine variation in the field. Conversely, artificial selection studies of physiological aspects of endocrine variation have not been conducted in as much detail as in insects.

Single locus polymorphisms in the field. In one of the first studies of a genetically variable endocrine trait in the field, Kallman (1989 and references therein) identified numerous alleles (five in one species) at the $P$ (pituitary) locus that segregate in natural populations of Xiphophorus (platyfish). $P$ alleles affect pituitary function strongly and male genotypes can differ substantially in adult size and the appearance of male secondary sexual characteristics (e.g., male caudal appendage) in the laboratory. Although it is termed the pituitary locus, the actual site of action of the $P$ locus was traced to higher centers of the brain that involve the production, release, or fate of gonadotropin-releasing hormone (GnRH) (Halpern-Sebold et al. 1986, Kallman 1989). $\mathrm{GnRH}$ ultimately regulates the production of gonadal steroids (e.g., testosterone and estrogens) via effects on pituitary gonadotropins. Investigators conducted these genetic and endocrine studies during the 1970s and 1980s and unfortunately no additional endocrine work has been published on this system since then. Because both natural and sexual selection affect body size in Xiphophorus (Basolo \& Wagner, 2004), the $P$ locus may be an important factor in sexual selection and life history evolution in species of this genus in the field. The $P$ locus-body size system shows great promise in the integration of endocrine-physiological and life history microevolutionary studies in the field.

A number of studies investigated adaptive variation at the melanocortin receptor gene Mc1r in vertebrates, which mediates melanocyte activity and body color. Researchers identified associations between Mc1r DNA sequence and body-color phenotype in some field populations of mice and lizards (Nachman et al. 2003, Rosenblum et al. 2004) and in several bird species (Mundy et al. 2003, Theron et al. 2001). Non- 
synonymous mutations at different sites of the Mc1r locus during the Pleistocene appear to have given rise to independently evolved melanic plumage polymorphisms in geese and skuas (Mundy et al. 2003). Results from molecular population genetic and association analyses support the hypothesis that selection has acted on Mc1r in the little striped whiptail (Aspidoscelis inornata) (Rosenblum et al. 2004). More recently, Hoekstra and coworkers (2006) reported that the Mc1r gene in extremely light-colored beach mouse populations of Peromyscus polionotus differs by a single, derived, chargechanging amino acid compared with McIr from more darkly colored mainland populations. Importantly, Hoekstra and coworkers (2006) reported that the amino-acid substitution changed the binding characteristics of the receptor. To our knowledge, this is the first direct demonstration in natural populations of adaptive genetic variation in receptor function.

Colosimo and coworkers (2005) investigated the role of DNA sequence variation in the Ectodysplasin ( $E d a)$ gene in the context of the evolution of reduced bony armor in the three-spined stickleback, Gasterosteus aculeatus, an extensively studied model in evolutionary and ecological genetics. The Eda gene encodes a secreted, locally acting signaling molecule (paracine regulator) that influences the development of dermal bones. Parallel evolution of the low-plated phenotype found in freshwater environments appears to have resulted from repeated selection on Eda alleles, derived from an ancestral haplotype, that are present in low frequencies in marine populations.

Artificial selection studies. Researchers performed several artificial selection studies of vertebrate endocrine traits in an evolutionary-behavioral context. In Japanese quail (Coturnix coturnix japonica), selection for low or high stress-induced cortico-sterone secretion led to changes in behavioral phenotype in the high stress line (e.g., greater avoidance, more fear-related behavior, and higher plasma corticosterone release in response to capture and restraint), but did not affect baseline corticosterone levels (Jones et al. 1994). A similar response occured for zebra finches selected directly on stress-induced corticosterone levels (Evans et al. 2006). These lines, together with testosterone titer manipulation, are in use to dissect the relative contributions of corticosterone and testosterone to the trade-off between immune function and the development of sexual signals (Roberts et al. 2007). Other studies involved selection on putative endocrine-mediated traits and investigated the correlated response of endocrine traits (mainly hormone titers). In a carefully controlled artificial selection study, Garland (Garland 2003) measured the titers of several hormones (e.g., corticosterone and leptin) in lines of the house mouse divergently selected for voluntary wheel-running (Malisch et al. 2007 and references therein). Ongoing research is aimed at quantifying levels of the corticosterone receptor and its binding protein in selected and control lines (T.G. Garland Jr., personal communication). In great tits (Parus major), divergent selection on personality produced lines of less aggressive, more cautious birds (slow explorers), and more aggressive fast explorers. Slow explorers showed a greater hypothalamic-pituitary-adrenal axis reactivity to social challenge compared with fast explorers (Carere et al. 2003, Groothuis \& Carere 2005).

Hoeflich and coworkers (2004) found that high-growth lines of mice derived from several independent artificial selection experiments consistently exhibited elevated 
serum levels of insulin growth factor 1 (IGF1) but not growth factor binding proteins, nor elevated expression of IGF receptors in muscle. This is a unique study in that it compares the contribution of variable genes that encode plasma hormone levels versus hormone receptors to the response to selection in a key life history trait. The findings of Hoeflich and coworkers (2004) support the strong association between a specific IGF1 haplotype and size in dog breeds (Sutter et al. 2007), which suggests that IGF1 was also an endocrine target of selection on size during the early history of dog domestication.

Fitness correlates of endocrine variation in the field. Topics of central importance in evolutionary endocrinology are the extent to which endocrine genetic variation gives rise to variation in fitness in the field and the mechanisms involved (Ketterson et al. 1996). Several long-term field studies in vertebrates used hormonal and other physiological manipulations to address these topics. In a series of pioneering studies, Sinervo and colleagues (Sinervo 1999, Sinervo et al. 2006, Svensson \& Sinervo 2004, and references therein) manipulated follicle-stimulating hormone (FSH) and follicle size to investigate the endocrine mechanisms that control the egg size/egg number tradeoff. The same group used HM to study fitness effects of endocrine-mediated variation such as the trade-off between fecundity and offspring survival (Sinervo \& Doughty 1996) and the cost of reproduction (Sinervo \& DeNardo 1996; for a similar study in mammals see Oksanen et al. 2002). These authors proposed very specific endocrine regulatory mechanisms that underlie these trade-offs. For example, genetically based covariation between egg and clutch size is thought to arise from variation in synthesis, hormone titers, or receptor sensitivity for GnRH and FSH, and corticosterone may regulate female condition, immune function, and fecundity (Sinervo \& Calsbeek 2003, Svensson et al. 2002). However, to date few components of hormonal regulation have been quantified directly (in some cases owing to a lack of appropriate hormone assays) and the extent to which analogous, natural hormonal variation regulates these tradeoffs in unmanipulated lizards has yet to be established. In addition, the same manipulations that Sinervo (1999) used do not always generate consistent changes in egg and clutch size in other taxa (Christians \& Williams 2002, Ji \& Diong 2006).

In a very thorough study, Ketterson and colleagues (Ketterson et al. 1996, 2001) used HM to elevate plasma testosterone (T) in male dark-eyed juncos (Junco hyemalis) to determine the selection pressures that may have shaped the typical distribution of hormone titers in natural populations. Validation studies showed that T-implants cause a prolonged elevation of plasma $\mathrm{T}$ within the normal physiological range and this in turn affects many different phenotypic traits, presumably reflecting the pleiotropic actions of T. Compared with control males, high-T males have higher song rates, larger territory size, are more attractive to females, and gain more extrapair fertilizations, but they also show decreased parental behaviors (less nest defense, lower chick feeding rates) and have lower survival, perhaps related to lower body fat, higher plasma corticosterone, suppressed immune function, and delayed molt (Ketterson et al. 1996, 2001). In a recent paper, Reed and coworkers (2006) combined the long-term HM data from these studies with population modeling to test explicitly the prediction that experimentally manipulated high-T males (the extreme phenotype) have lower fitness than control 
males (where plasma T levels were shaped by selection). Surprisingly, they found the opposite: High-T males have higher fitness than control males owing to higher rates of extrapair copulation by $\mathrm{T}$ males. These authors suggested that testosterone levels may be constrained in natural populations, although there are other potential explanations such as selection via indirect effects of $T$ on offspring or females (Reed et al. 2006). A major unanswered question generated by these studies is the extent to which experimental manipulation mimics the pleiotropic effects of segregating genetic variation for endocrine regulators in these and other species.

\section{Summary and Conclusions}

During the past two decades, the first direct information on the extent, characteristics, and functional significance of genetic variation for endocrine traits in nondomesticated animals was obtained using well-validated endocrine techniques. This is the most important achievement of the nascent field of evolutionary endocrinology thus far. Insect and vertebrate studies contributed in complementary ways to our understanding of evolutionary endocrinology: Insect studies provided the most detailed information on the endocrine-genetics of hormone titers, titer regulators, and intracellular signaling in the laboratory; vertebrate studies contributed the most to our understanding of the endocrine basis of single-locus polymorphisms and fitness effects of endocrine variation in the field.

Results to date clearly implicate variation in endocrine regulation as an important and widespread aspect of organismal microevolution. Prominent examples include morphs of complex polymorphisms in insects (dispersal and wing-pattern polymorphisms in crickets and butterflies) and vertebrates (size and plate-morph polymorphisms in fish; color polymorphisms in various species). Genetic variation in endocrine regulation may play an important role in both developmental and reproductive aspects of these ecologically important polymorphisms. Similarly, mutational analysis implicates the modulation of insulin signaling in the microevolution of individual and suites of life history traits in D. melanogaster.

In spite of marked progress, evolutionary endocrinology is in its infancy, and studies of endocrine variation and microevolution have only scratched the surface of the mechanisms involved. Most empirical studies have focused on the physiological-genetic aspects of systemic hormone titers and titer regulators of only a few hormones (juvenile hormone and ecdysteroids in insects; testosterone and corticosterone in vertebrates), measured in a handful of species, under a limited number of environmental conditions. For example, only one study has investigated circadian aspects of endocrine genetic variation, although such influences may be widespread, and may substantially complicate the interpretation of endocrine data if not identified (Zera \& Cisper, 2001, Zhao \& Zera 2004). Thus, a major goal of evolutionary endocrinology in the next decade should be to obtain direct and detailed measurements of titers and titer regulators on these and additional hormones. Unless this is done, it will not be possible to determine the extent to which results obtained to date represent general endocrine adaptations. 
The next decade will see the considerable expansion of evolutionary endocrinology because key topics that have largely been ignored will almost certainly be the focus of intense study. Most notable will be studies of genetic variation in hormone receptors and signaling pathways and their role in organismal adaptation. Investigations of these topics have thus far been hampered by the lack of appropriate molecular tools; however, model organisms for molecular-genetic studies are being used increasingly to address these issues (Richard et al. 2005), and molecular techniques are becoming available for a wider range of organisms (Colosimo et al. 2005, Hoekstra et al. 2006). Data on hormone receptor variation will be critically important for assessment of the relative occurrence of endocrine alterations that give rise to localized, organ- or tissue-specific changes in phenotypic expression (Hoeflich et al. 2004). Indeed, a key topic for future research is the relative occurrence of endocrine adaptations that involve alterations in systemic (titers) versus localized (receptor) regulators, the factors that select for one type of adaptation or another, and functional constraints that limit the evolution of a particular type of endocrine adaptation.

Although a number of classic studies in vertebrates investigated fitness consequences of endocrine-mediated phenotypic variation (FSH-mediated egg size/number trade-offs; testosterone effects on life history trade-offs in juncos), these studies investigated almost exclusively variation produced by hormonal manipulation. Genetic variation has not been studied for these endocrine traits in unmanipulated individuals. Thus, the relevance of the manipulation studies to both the existence and fitness effects of natural endocrine genetic variation remains to be established. More integrative studies are needed in which detailed investigation of the endocrine-genetic correlates of phenotypic variation is combined with experimental manipulations performed in the appropriate ecological context.

In conclusion, new molecular techniques are beginning to allow investigation of important but previously unapproachable endocrine issues such as variation in receptor expression. However, as exciting as these developments are, studies which focus solely on the identification of candidate genes with overexpression studies or mutational analyses of single protein products will not fully describe the complexity and integrated nature of endocrine regulatory networks. Rather, molecular and biochemical studies of receptor expression, receptor function, and intracellular signaling must be integrated with classical approaches, which involve detailed measurement of hormone titers and titer regulators in quantitative-genetics experiments. Moreover, experimental manipulations must be incorporated to assess the functional significance of endocrine variation at both the whole organism and molecular levels, and ideally in some cases these studies will be integrated with fitness studies conducted under appropriate ecological conditions. These are exciting times for evolutionary endocrinology, which is beginning to contribute significantly to our understanding of the evolution of complex adaptations. 


\section{Acknowledgments}

A.J.Z. acknowledges support from NSF (most recently, IBN-9507388, IBN 0130665, and IBN-0212486), and L.G.H. acknowledges support from NIH (NIDDK, 1R01 DK074136-01) and NSF (EPS-0346476). T.D.W. was supported by a Discovery Grant from the Natural Sciences and Engineering Research Council of Canada. We thank J. Christian for comments on an earlier draft of the manuscript.

\section{Literature Cited}

Adkins-Regan E. 2005. Hormones and Animal Social Behavior. Princeton: Princeton Univ. Press. 429 pp.

Baker FC. 1990. Techniques for identification and quantification of juvenile hormones and related compounds in arthropods. In Morphogenetic Hormones of Arthropods, Vol. 1, ed. AP Gupta, pp. 389-453. New Brunswick: Rutgers Univ. Press

Basolo A, Wagner W. 2004. Covariation between predation risk, body size and fin elaboration in the green swordtail, Xiphophorus helleri. Biol. J. Linn. Soc. 83:87-100

Bohni R, Riesgo-Escovar J, Oldham S, Brogiolo W, Stocker H, et al. 1999. Autonomous control of cell and organ size by CHICO, a Drosophila homolog of vertebrate IRS. Cell 97:865-75

Brakefield P, Frankino W. 2007. Polyphenisms in Lepidoptera: Multidisciplinary approaches to studies of evolution and development. In Phenotypic Plasticity in Insects: Mechanisms and Consequences, ed. T Ananthakrishnan, D Whitman. pp. 121-152. Science Publishers

Brakefield P, French V, Zwaan B. 2003. Development and the genetics of evolutionary change within insect species. Annu. Rev. Ecol. Syst. 34:633-60

Breuner CW, Orchinik M. 2002. Beyond carrier proteins: plasma binding proteins as mediators of corticosteriod action in vertebrates. J. Endocrinol. 175:99-112

Carere C, Groothuis T, Mostl E, Dann S, Koolhaus J. 2003. Fecal corticosteroids in a territorial bird selected for different personalities: daily rhythm and response to social stress. Horm. Behav. 43:540-48

Christians JK, Williams TD. 2002. Organ mass dynamics in relation to yolk production and egg formation in European starlings Sturnus vulgaris. Physiol. Biochem. Zool. 72:455-61

Clancy D, Gems D, Harshman LG, Oldham SH, Stocker H, et al. 2001. Extension of lifespan by loss of CHICO, a Drosophila insulin receptor substrate protein. Science 292:104-6

Colon J, Larhammar D. 2005. The evolution of neuroendocrine peptides. Gen. Comp. Endocrinol. 142:53-59

Colosimo P, Hosemann K, Balabhadra S, Villarreal G, Dickson M, et al. 2005. Widespread parallel evolution in sticklebacks by repeated fixation of ectodysplasin alleles. Science 307:1928-33 
Crone E, Zera AJ, Anand A, Oakeshott J, Sutherland T, et al. 2007. JHE in Gryllus assimilis: Cloning, sequence-activity associations and phylogeny. Insect Biochem. Molec. Biol. In press

D’Amico L, Davidowicz G, Nijhout HF. 2001. The developmental and physiological basis of body size evolution in an insect. Proc. R. Soc. London Ser. B 268:1589-93

Davidowicz G, D’Amico L, Nijhout HF. 2003. Critical weight in the development of insect body size. Evol. Dev. 5:188-97

Davidowicz G, Nijhout HF. 2004. The physiological basis of reaction norms: The interaction among growth rate, the duration of growth and body size. Integr. Comp. Biol. 44:443-49

De Jong G, Bochdanovits Z. 2003. Latitudinal clines in Drosophila melanogaster: body size, allozyme frequencies, inversion frequencies, and the insulin signaling pathway. J. Genet. 82:207-23

Dingle H, Winchell R. 1997. Juvenile hormone as a mediator of plasticity in insect life histories. Arch. Insect Biochem. Physiol. 35:359-73

Drummond-Barbarosa D, Spradling A. 2001. Stem cells and their progeny respond to nutritional changes during Drosophila oogenesis. Dev. Biol. 231:265-78

Edgar B. 2006. How flies get their size: genetics meets physiology. Nat. Rev. Genet. 7:907-16

Emlen DJ, Nijhout HF. 1999. Hormonal control of male horn length dimorphism in the dung beetle Onthophagus taurus (Coleoptera: Scarabaeidae). J. Insect Physiol. 45:45-53

Emlen DJ, Nijhout HF. 2001. Hormonal control of male horn length dimorphism in Onthophagus taurus (Coleoptera: Scarabaeidae): a second critical period of sensitivity to juvenile hormone. J. Insect Physiol. 47:1045-54

Emlen DJ, Szafran Q, Corley L, Dworkin I. 2006. Insulin signaling and limb-patterning candidate pathways for the origin of evolutionary diversification. Heredity 97:179-91

Evans M, Roberts M, Buchanan K, Goldsmith A. 2006. Heritability of corticosterone responses and changes in life history traits during selection in the zebra finch. J. Evol. Biol. 19:343-52

Falconer DS, Mackay TF. 1996. Introduction to Quantitative Genetics, 4th ed. Essex: Longman. 464 pp.

Feder ME, Bennett AF, Burggren WW, Huey RB. 1987. New Directions in Ecological Physiology. Cambridge: Cambridge Univ. Press. 364 pp.

Finch CE, Rose MR. 1995. Hormones and the physiological architecture of life history evolution. Q. Rev. Biol. 70:1-51

Flatt T, Tu M-P, Tatar M. 2005. Hormonal pleiotropy and the juvenile hormone regulation of Drosophila development and life history. BioEssays 27:999-1010

Garland T Jr. 2003. Selection experiments: an underutilized tool in biomechnics and organismal biology. In Vertebrate Biomechanics and Evolution, ed. V Bels, J-P Gasc, A Casinos, pp. 23-65. Oxford: BIOS Scientific 
Garland TJ Jr, Carter PA. 1994. Evolutionary physiology. Annu. Rev. Physiol. 56:579-621

Gibbs AG. 1999. Laboratory selection for the comparative physiologist. J. Exp. Biol. 202:2709-18

Gilbert LI, Iatrou K, Gill S, eds. 2005. Comprehensive Molecular Insect Science, Vol 3. Endocrinology. Amsterdam: Elsevier

Groothuis T, Carere C. 2005. Avain personalities: characterization and epigenesis. Neurosci. Biobehav. Rev. 29:137-50

Gu X, Zera AJ. 1996. Quantitative genetics of juvenile hormone esterase, juvenile hormone binding and general esterase activity in the cricket, Gryllus assimilis. Heredity 76:136-42

Guilgur L, Moncaut N, Canario A, Somoza G. 2006. Evolution of GnRH ligands and receptors in gnathostomata. Comp. Biochem. Physiol. 144A:272-83

Halpern-Sebold L, Schreibman M, Margolis-Nunno H. 1986. Differences between earlyand late-maturing genotypes of the platyfish (Xiphophorus maculatus) in the morphology of their immunoreactive lutenizing hormone releasing hormone-containing cells: A developmental study. J. Exp. Zool. 240:245-57

Harshman LG, Hoffmann AA. 2000. Laboratory selection experiments using Drosophila: what do they really tell us? Trends Ecol. Evol. 15:32-36

Harshman LG, Zera A. 2007. The cost of reproduction: the devil in the details. Trend. Ecol. Evol. 22:80-86

Hartfelder K, Emlen DJ. 2005. Endocrine control of insect polyphenism. See Gilbert et al. 2005, pp. 651-703

Henrich VC. 2005. The ecdysteroid receptor. See Gilbert et al. 2005, pp. 243-85

Hoekstra HE, Hirschmann RJ, Bundey RA, Insel PA, Crossland JP. 2006. A single amino acid mutation contributes to adaptive beach mouse color pattern. Science 313:101-4

Hoeflich A, Bunger L, Nedbal S, Renne U, Elmlinger MW, et al. 2004. Growth in mice reveals conserved and redundant expression patterns of the insulin-like growth factor system. Gen. Comp. Endocrinol. 136:248-59

Ji X, Diong C-H. 2006. Does follicle excision always result in enlargement of offspring size in lizards? J. Comp. Physiol. 176:521-25

Jones G, Jones, D. 2007. Farnesoid secretions of dipteran ring glands: what we do know and what we can know. Insect Biochem. Mol. Biol. 37:771-98

Jones M, Rakes L, Yochum M, Dunn G, Wurster S, et al. 2006. The proximate control of pupal color in swallowtail butterflies: Implications for the evolution of environmentally cued pupal color in butterflies (Lepidoptera: Papilionidae). J. Insect Physiol. 53:40-46.

Jones R, Satterlee D, Ryder F. 1994. Fear of humans in Japanese quail selected for low and high adrenocortical response. Physiol. Behav. 56:379-83

Kacsoh B. 2000. Endocrine Physiology. New York: McGraw-Hill. 739 pp.

Kallman K. 1989. Genetic control of size at maturity in Xiphophorus. In Ecology and Evolution of Livebearing Fishes (Peociliidae), ed. G Meffe, F Snelson Jr, pp. 163-84. Englewood Cliffs: Prentice-Hall 
Ketterson E, Nolan V Jr,Castro J, Buerkle C, Clotfelter E, et al. 2001. Testosterone, phenotype, and fitness: a research program in evolutionary behavioral endocrinology. In Avian Endocrinology, ed. A Dawson, C Chaturvedi, pp. 19-40. New Delhi, India: Narosa Publishing House

Ketterson E, Nolan V Jr, Cawthorn M, Parker P, Ziegenfus C. 1996. Phenotypic engineering: using hormones to explore the mechanistic and functional bases of phenotypic variation in nature. Ibis 138:70-86

King R, Cline J, Hubbard C. 2004. Heritable variation in testosterone levels in male garter snakes (Thamnophis sirtalis). J. Zool. Lond. 264:143-47

Koch P, Brakefield P, Kesbeke F. 1996. Ecdysteroids control eyespot size and wing colour pattern in the polyphenic butterfly, Bicyclus anynana (Lepidoptera: Satyridae). J. Insect Physiol. 42:223-30

Malisch J, Salzman W, Gomes F, Rezende E, Jeske D, Garland T Jr. 2007. Baseline and stress-induced plasma corticosterone concentrations of mice selectively bred for high voluntary wheel running. Physiol. Biochem. Zool. 80:146-56

Marler CA. 1988. Evolutionary costs of aggression revealed by testosterone manipulations in free-living male lizards. Behav. Ecol. Sociobiol. 23:21-26

Matsuda R. 1987. Animal Evolution in Changing Environments with Special Reference to Abnormal Metamorphosis. New York: Wiley-Interscience. 355 pp.

Meylan S, Clobert J. 2005. Is corticosterone-mediated phenotype development adaptive? Maternal corticosterone treatment enhances survival in male lizards. Horm. Behav. 48:44-52

Moczek A, Nijhout HF. 2002. Developmental mechanisms of threshold evolution in a polyphenic beetle. Evol. Dev. 4:252-64

Mundy NI, Badcock NS, Hart T, Scribner K, Janssen K. 2003. Conserved genetic basis of a quantitative plumage trait involved in mate choice. Science 303:1870-73

Nachman M, Hoekstra H, D'Augustino S. 2003. The genetic basis of adaptive melanism in pocket mice. Proc. Natl. Acad. Sci. USA 100:5268-73

Nijhout HF. 1991. The Development and Evolution of Butterfly Wing Patterns. Washington and London: Smithsonian Institution Press. 336 pp.

Nijhout HF. 1994. Insect Hormones. Princeton: Princeton Univ. Press. 267 pp.

Nijhout HF. 2003. The control of body size in insects. Dev. Biol. 261:1-9

Nijhout HF, Williams C. 1974. Control of moulting and metamorphosis in the tobacco hornworm, Manduca sexta (L.): cessation of juvenile hormone secretion as a trigger for pupation. J. Exp. Biol. 61:493-501

Nijhout HF, Wheeler D. 1982. Juvenile hormone and the physiological basis of insect polymorphism. Q. Rev. Biol. 57:109-33

Norris DO. 1997. Vertebrate Endocrinology. San Diego: Academic. 634 pp.

Oksanen T, Koskela E, Mappes T. 2002. Hormonal manipulation of offspring number: maternal effort and reproductive costs. Evolution 56:1530-37 
Partridge L, Gems D, Withers DJ. 2005. Sex and death: what is the connection? Cell 120:461-72.

Reed WL, Clark ME, Parker PG, Raoulf SA, Arguedas N, et al. 2006. Physiological effects on demography: A long-term experimental study of testosterone's effects on fitness. Am. Nat. 167:667-83

Richard D, Rybczynski R, Wilson T, Wang Y, Wayne M, et al. 2005. Insulin signaling is necessary for vitellogenesis in Drosophila melanogaster independent of the roles of juvenile hormone and ecdysteroids: female sterility of the chico ${ }^{1}$ insulin signaling mutation is autonomous to the ovary. J. Insect Physiol. 51:455-64

Roberts M, Buchanan K, Hasselquist D, Evans M. 2007. Effects of testosterone and corticosterone on immunocompetence. Horm. Behav. 51:126-34

Roff DA. 1996. The evolution of threshold traits in animals. Q. Rev. Biol. 71:3-35

Roff DA, Sterling G, Fairbairn DJ. 1997. The evolution of threshold traits: a quantitative genetic analysis of the physiological and life-history correlates of wing dimorphism in the sand cricket. Evolution 51:1910-19

Rosenblum E, Hoekstra H, Nachman M. 2004. Adaptive reptile color variation and the evolution of the Mc1r gene. Evolution 58:1794-808

Rountree DB, Nijhout HF. 1995a. Genetic control of a seasonal morph in Precis coenia (Lepidoptera: Nymphalidae). J. Insect Physiol. 41:1141-45

Rountree DB, Nijhout HF. 1995b. Hormonal control of a seasonal polyphenism in Precis coenia (Lepidoptera: Nymphalidae). J. Insect Physiol. 41:987-92

Rybczynski R. 2005. Prothoracicotropic hormone. See Gilbert et al. 2005, pp. 61-123

Shi Y-B. 2000. Amphibian metamorphosis. From Morphology to Molecular Biology. Wiley-Liss, 288 pp.

Shingleton AW, Das J, Vinicius L, Stern DL. 2005. The temporal requirements for insulin signaling during development in Drosophila. PLoS Biol. 3:1607-17

Sinervo B. 1999. Mechanistic analysis of natural selection and a refinement of Lack's and Williams's principles. Am. Nat. 154:S26-42

Sinervo B, Calsbeek R. 2003. Physiological epistasis, ontogenetic conflict and natural selection on physiology and life history. Integr. Comp. Biol. 43:419-30

Sinervo B, DeNardo D. 1996. Costs of reproduction in the wild: path analysis of natural selection and experimental tests of causation. Evolution 50:1299-313

Sinervo B, Doughty P. 1996. Interactive effects of offspring size and timing of reproduction on offspring reproduction: experimental, maternal and quantiative genetics aspects. Evolution 50:1314-27

Sinervo B, Chaine A, Clobert J, Calsbeek R, Hazard L, et al. 2006. Self-recognition, color signals, and cycles of greenbeard mutualism and altruism. Proc. Natl. Acad. Sci. USA 103:7372-77

Solberg L, Baum A, Ahmadiyeh N, Shimomura K, Li R, et al. 2006. Genetic analysis of the stress-responsive adrenocortical axis. Physiol. Genomics 27:362-69 
Stern DL. 2000. Perspective: Evolutionary developmental biology and the problem of variation. Evolution 54:1079-91

Sutter NB, Bustamante CD, Chase K, et al. 2007. A single IGF1 allele is a major determinant of small size in dogs. Science 316:112-15

Suzuki Y, Nijhout HF. 2006. The evolution of a polyphenism by genetic accomodation. Science 311:650-52

Svensson E, Sinervo B. 2004. Spatial scales and temporal component of selection in sideblotched lizards. Am. Nat. 163:726-34

Svensson E, Sinervo B, Comendant T. 2002. Mechanistic and experimental analysis of condition and reproduction in a polymorphic lizard. J. Evol. Biol. 15:1034-47

Swallow J, Garland T Jr. 2005. Selection experiments as a tool in evolutionary and comparative physiology: insights into complex traits - an introduction to the symposium. Integr. Comp. Biol. 45:387-90

Tatar M, Kopelman A, Epstein D, Tu M-P, Yin C-M, Garofalo RS. 2001. A mutant Drosophila insulin receptor homolog that extends life-span and impairs neuroendocrine function. Science 292:107-10

Theron E, Hawkins K, Bermingham E, Ricklefs R, Mundy NI. 2001. The molecular basis of an avian plumage polymorphism in the wild. A melanocortin-1-receptor point mutation is perfectly associated with the melanic plumage morph of the bananaquit, $\mathrm{Co}_{0}$ ereba flaveola. Curr. Biol. 11:550-57

Tu M, Yin C-M, Tatar M. 2002. Impared ovarian ecdysone synthesis of Drosophila melanogaster insulin receptor mutants. Aging Cell. 1:158-60

Tu M-P, Yin C-M, Tatar M. 2005. Mutations in insulin signaling pathway alter juvenile hormone synthesis in Drosophila melanogaster. Gen. Comp. Endocrinol. 142:347-56

Vafopoulou X, Steel CGH. 2005. Circadian organization of the endocrine system. See Gilbert et al. 2005, pp. 551-650

West-Eberhard M. 2003. Developmental Plasticity and Evolution. Oxford: Oxford Univ. Press. 816 pp.

Zera AJ. 2004. The endocrine regulation of wing polymorphism: State of the art, recent suprises, and future directions. Integr. Comp. Biol. 43:607-16

Zera AJ. 2006. Evolutionary genetics of juvenile hormone and ecdysteroid regulation in Gryllus: A case study in the microevolution of endocrine regulation. Comp. Biochem. Physiol. 144A:365-79

Zera AJ. 2007a. Wing polymorphism in Gryllus (Orthoptera:Gryllidae): Endocrine, energetic and biochemical bases of morph specializations for flight vs reproduction. In Phenotypic Plasticity in Insects: Mechanisms and Consequences, ed., T Ananthakrishnan, D Whitman, pp. 547-90. Plymouth, United Kingdom: Sci. Publ.

Zera AJ. 2007b. Endocrine analysis in evolutionary-developmental studies of insect polymorphism: Use and misuse of hormone manipulation. Evol. Dev. 9:499-513 
Zera AJ, Cisper G. 2001. Genetic and diurnal variation in the juvenile hormone titer in a wing-polymorphic cricket: Implications for the evolution of life histories and dispersal. Physiol. Biochem. Zool. 74:293-306

Zera AJ, Harshman LG. 2001. Physiology of life history trade-offs in animals. Annu. Rev. Ecol. Syst. 32:95-126

Zera AJ, Holtmeier CL. 1992. In vivo and in vitro degradation of juvenile hormone-III in presumptive long-winged and short-winged Gryllus rubens. J. Insect Physiol. 38:61-74

Zera AJ, Huang Y. 1999. Evolutionary endocrinology of juvenile hormone esterase: Functional relationship with wing polymorphism in the cricket, Gryllus firmus. Evolution 53:837-47

Zera AJ, Sanger T, Cisper GL. 1998. Direct and correlated responses to selection on JHE activity in adult and juvenile Gryllus assimilis: implications for stage-specific evolution of insect endocrine traits. Heredity 80:300-9

Zera AJ, Strambi C, Tiebel KC, Strambi A, Rankin MA. 1989. Juvenile hormone and ecdysteroid titers during critical periods of wing morph determination in Gryllus rubens. J. Insect Physiol. 35:501-11

Zera AJ, Tiebel KC. 1989. Differences in juvenile hormone esterase activity between presumptive macropterous and brachypterous Gryllus rubens: Implications for the hormonal control of wing polymorphism. J. Insect Physiol. 35:7-17

Zera AJ, Zhang C. 1995. Direct and correlated responses to selection on hemolymph juvenile hormone esterase activity in Gryllus assimilis. Genetics 141:1125-34

Zera AJ, Zhao Z, Kaliseck K. 2007. Hormones in the field: Evolutionary endocrinology of juvenile hormone and ecdysteroids in field populations of the wing-dimorphic cricket Gryllus firmus. Physiol. Biochem. Zool. In press

Zhao Z, Zera AJ. 2004. The hemolymph JH titer exhibits a large-amplitude, morph-dependent, diurnal cycle in the wing-polymorphic cricket, Gryllus firmus. J. Insect Physiol. 50:93-102

Zijlstra W, Steigenga M, Koch P, Zwan B, Brakefield P. 2004. Butterfly selected lines explore the hormonal basis of interactions between life histories and morphology. Am. Nat. 163:E76-87 